\title{
Analysis of Shooting Consistency in Archers: A Dynamic Time Warping Algorithm-Based Approach
}

\author{
Cheng-Hao Quan, ${ }^{1}$ Zia Mohy-Ud-Din, ${ }^{2}$ and Sangmin Lee ${ }^{1,3}$ \\ ${ }^{1}$ Institute for Information and Electronic Research (IIER), Inha University, Incheon 22212, Republic of Korea \\ ${ }^{2}$ Department of Biomedical Engineering, Sir Syed University of Engineering and Technology, Karachi, Pakistan \\ ${ }^{3}$ Department of Electronic Engineering, Inha University, Incheon 22212, Republic of Korea
}

Correspondence should be addressed to Sangmin Lee; sanglee@inha.ac.kr

Received 25 October 2017; Accepted 2 December 2017; Published 31 December 2017

Academic Editor: Ka L. Man

Copyright (c) 2017 Cheng-Hao Quan et al. This is an open access article distributed under the Creative Commons Attribution License, which permits unrestricted use, distribution, and reproduction in any medium, provided the original work is properly cited.

\begin{abstract}
The shooting consistency of an archer is commonly perceived to be an important determinant of successful scores. Four $(n=4)$ elementary level archers from a middle school in Korea participated in this study. In order to quantify shooting consistency, movement of the bow forearm was measured with an inertia sensor during archery shooting. The shooting consistency was calculated and defined by the dynamic time warping (DTW) algorithm as the distance between two time sequences of acceleration data. Small distance values indicate that the archer has maintained high-level shooting consistency while archery shooting repetitively. To verify the shooting consistency metric, the relationship between scores and shooting consistency is evaluated. The results show that the higher the scores achieved by the archer, the higher is the level of shooting consistency demonstrated.
\end{abstract}

\section{Introduction}

Recent advances in microelectromechanical system (MEMS) technologies and wireless communication have allowed the development of small low-cost inertia sensors that can measure body movements precisely and conveniently. The use of inertia sensors to measure activity levels for sporting is emerging as a popular method for the biomechanical quantification of sporting activities (e.g., archery shooting).

Few studies on postural consistency or movement during archery shooting have been found in the literature. To the best of our knowledge, the first researchers to pay attention to postural consistency were Stuart and Atha. In their study [1], Stuart and Atha used a three-dimensional optoelectronic scanner to measure the positions of the archer's head and elbow and that of the bow at the moment of "loose" (releasing the arrow). Postural consistency is defined as the consistency in the archer's positioning that is within-ends the standard deviation (SD) of the positions for each archer.

More recent studies [2,3] have reported considerable findings with regard to the archer's movement during arrow release. In [2], by comparing the muscular activation patterns of the forearm that manipulates the bow ("bow-forearm") of elite archers and beginners, the study concluded that the action of not contracting both $\mathrm{M}$. flexor digitorum superficialis (MFDS) and M. extensor digitorum (MED), or of only contracting MED, during archery shooting is an indicator of the archer's performance level. In [3], by examining the finger and bowstring movements during arrow release, the study presumed that maximum lateral bowstring deflection does not adversely affect the archer's performance. However, the two aforementioned studies used EMG data to analyze muscular activation patterns or camera-based motion tracking systems to quantify movement; furthermore, the movement or muscular activation only focused on the release moment.

Motion analysis of the movements performed individually by an archer in training or competition provides information about the correctness of the individual techniques and the effectiveness of the archer's strategies. Currently, there are motion tracking systems that use inertia sensors and do not need cameras or stick markers, and they can 


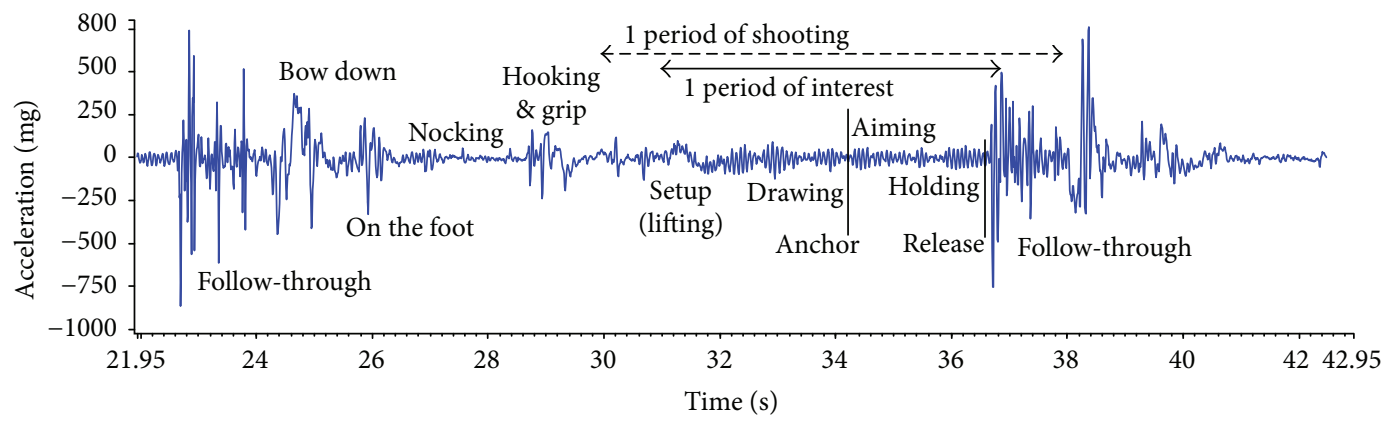

FIGURE 1: Example of acceleration data (21.95 s-42.95 s): measured bow-forearm movement ( $x$-axis: drawing direction) during successive archery shootings (sampling rate of $100 \mathrm{~Hz}$ ).

be used anywhere. Such systems also make it possible to analyze body movements during archery shooting more precisely and conveniently than before. The disadvantage is the high cost and need to properly process and interpret measured data.

In this study, we measure bow-forearm movement by using inertia sensors during archery shooting to evaluate the shooting consistency of archers. Moreover, we attempt to provide movement analysis tools that work precisely and conveniently. Shooting consistency is defined as a function of the dynamic time warping (DTW) distance between two time sequences of acceleration data calculated with the DTW algorithm. Small distance values indicate that an archer has maintained high-level shooting consistency while archery shooting repetitively. To the best of our knowledge, no quantifying shooting consistency in archery by using inertia sensors has been reported in the literature. Here, we present the DTW algorithm as a useful signal processing method for quantifying shooting consistency in archery.

\section{Materials and Methods}

2.1. Archery Shooting. Archery can be described as a comparatively static sport that requires strength and endurance of the upper body, in particular, the forearm and shoulder girdle [4]. Skill in archery is defined as the ability to shoot an arrow onto a given target in a certain time span with accuracy [5]. Archery shooting is described as a three-phase (drawing, aiming, and release) movement. Alternatively, Nishizono et al. [6] further divided the stages of a shot into six: bow hold, drawing, full draw, aiming, release, and follow-through. An archery shot must be well balanced and highly reproducible in order to achieve commendable results in an archery competition.

Figure 1 shows an example of acceleration data that measures the bow-forearm movement during successive archery shootings. As shown in Figure 1, a one-shot period usually consists of nocking, hooking and grip, setup (bow lifting), and drawing that includes anchor moment, aiming, and holding, which in turn includes the release moment, follow through, and finish phases. Not all the phases can be separated exactly, but the phases are meaningful for training.

2.2. Measurement of Acceleration Data. Four elementary level (performance level: beginner, elementary, national, and world-class archer) female participants (middle school student archers) were recorded during archery shooting. The archer's age, previous season's scores, and current scores are as follows: $16,59,59.5 ; 14,59,59.5 ; 15,55,53.5 ; 14,54$, 53.7. The participants were briefed on the purpose of this study and the related procedures.

The participants were required to use their own bow and arrows for measurement purposes and were tested at their school's outdoor tracks. An acceleration data logger (Myomotion, Noraxon Inc., USA) was used to obtain detailed information on body movement over a round (six shots per round required almost $110 \mathrm{~s}-140 \mathrm{~s}$ ). An inertia sensor mounted on the bow-forearm was used to measure movement during archery shooting at a sampling frequency of $100 \mathrm{~Hz}$. For each recording round, an archer shot six shots at a distance of $30 \mathrm{~m}$ and took a short rest. Each participant shot a total of 36 arrows. Finally, we collected a total of 144 data sets from the four participants $(n=4)$ (considering references $[1,3]$ ).

2.3. Period for Analysis. In this study, for the analysis of shooting consistency in archery, we set the period of interest (POI, see Figure 1) that only includes some interesting phases, as follows: setup (not the whole period), drawing, aiming, holding, and a period of dozens of milliseconds after release.

As MEMS technology has developed, the ability to measure body movements more precisely and conveniently than before has increased. For the analysis of shooting consistency in archery, we choose wider range of POI than previous works. Such that it provides more useful information compared with studies that have focused on release moments only.

2.4. Method for Analysis of Shooting Consistency. In order to analyze shooting consistency, the bow-forearm movement is measured during archery shooting, and the DTW distance between two time sequences of acceleration data is calculated using the DTW algorithm.

DTW is a well-known technique for finding an optimal nonlinear mapping between two given time sequences (e.g., acceleration data in archery shooting) under certain restrictions [7]. The objective of DTW is to compare two time sequences. The distance (DTW distance) between $X$ and $Y$ indicates how much similarity there is between them. 


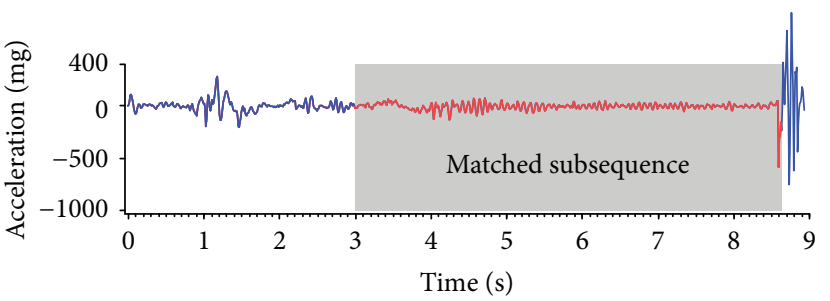

(a)

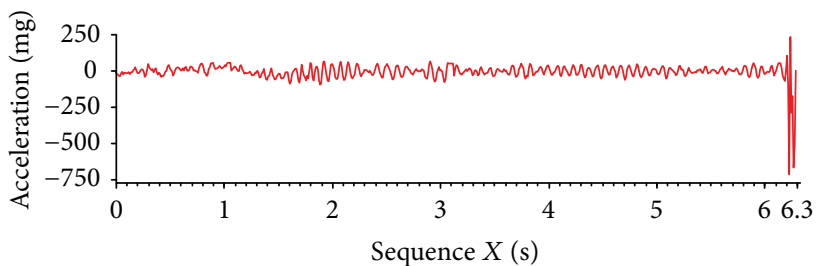

(b)

Figure 2: (a) Example of input sequences for DTW: query sequence $Y$ (109.21 s long) for one-round acceleration data and reference sequence $X$ (6.3 s long) within POI indicated by grey region. (b) Zoom-out of sequence that corresponds to sequence $X$ of (a).

In fields of speech recognition [8], and data mining and information retrieval [9-11], DTW has been successfully applied to cope with time deformations and different speeds associated with automatic time-dependent data.

In essence, DTW is an algorithm that can compute the similarity between two time sequences, even if the lengths do not match $[7,12]$. Assume that we have two time sequences, $X=\left(x_{1}, x_{2}, \ldots, x_{n}\right)$ of length $n \in N$ and $Y=\left(y_{1}\right.$, $\left.y_{2}, \ldots, y_{m}\right)$ of length $m \in N$. In order to measure the similarity between these two time sequences, we construct an $n$-by$m$ cost matrix where the $\left(i^{\text {th }}, j^{\text {th }}\right)$ element of the matrix contains cost $c\left(x_{i}, y_{j}\right)$ between two points $x_{i}$ and $y_{j}$. Typically, $c\left(x_{i}, y_{j}\right)$ is small (low cost) if $x_{i}$ and $y_{j}$ are similar to each other; otherwise, $c\left(x_{i}, y_{j}\right)$ is large (high cost). The cost matrix of $X$ and $Y$ using the Manhattan distance (absolute value of the difference) is a local cost measure $c$ (i.e., $\left.c\left(x_{i}, y_{j}\right)=\left|x_{i}-y_{j}\right|\right)$. An $(n, m-)$ warping path $p=\left(p_{1}, p_{2}, \ldots, p_{l}\right)$ of length $l \in N$ defines the mapping between the two time sequences $X$ and $Y$. The $k^{\text {th }}$ element of $p$ is defined as $p_{k}=(i, j)_{k}$. There are exponentially many warping paths. However, we are only interested in the path that minimizes the cost (warping cost) with respect to the local cost measure $c$, which is defined as the sum of all local costs, where $k$ runs from 1 to $l$. An optimal warping path between $X$ and $Y$ is a warping path $p^{*}$ with minimal total cost among all possible warping paths. The distance (DTW distance) $d(i, j)$ between $X$ and $Y$ is then defined as the total cost of $p^{*}$. This optimal warping path can be found efficiently by using dynamic programming to evaluate the following recursive steps: $d(i, j)=c(i, j)+\min$ $\{d(i-1, j-1), d(i-1, j), d(i, j-1)\}$. The value $d(i, j)$ defines an $n$-by- $m$ accumulated cost matrix. The initialization can be simplified by extending the accumulated cost matrix with an additional row and column and formally setting $d(i, 0)=\infty$ for $i \in[1: n], d(0, j)=\infty$ for $j \in[1: m]$, and $d(0,0)=0$. Furthermore, the optimal warping path $p^{*}=\left(p_{1}, p_{2}, \ldots, p_{l}\right)$ is computed in reverse order of the indices starting with $p_{l}=(n, m)_{l}$.

2.5. DTW with Finding Subsequences Automatically. In our study, DTW is used not only to compare two similar time sequences but also to find subsequences within the longer sequence that optimally fits the shorter sequence. The longer sequence represents a given one-round (six shots) acceleration data that we call "query sequence," and the shorter sequence represents a given acceleration data of
POI (see Figure 1) that we call "reference sequence." Before starting DTW, we select one sequence as the reference sequence where the archer achieved a score of 10 for shooting. The problem of finding the optimal subsequences can be solved by a variant of DTW that we call "aDTW" (DTW with finding subsequences automatically).

We illustrate the aDTW algorithm with the example described by figures. The input for aDTW consists of the query (sequence $Y$ in Figure 2(a)) and reference (sequence $X$ in Figures 2(a) and 2(b)).

In order to find a subsequence that minimizes the distance to the reference over all possible subsequences of query, we modify the initial conditions of the classic DTW algorithm by setting $d(i, 0)=\infty$ for $i \in[0: n]$ and $d(0, j)=0$ for $j \in[0: m]$. In the first iteration, we compute the accumulated cost matrix shown in Figure 3(a) and obtain the distance function (Figure 3(b)) that corresponds to the top row of the matrix.

By setting the threshold manually for each participants, we obtain the six local minima with distance below the threshold. The indices of the six local minima correspond to the end point of the six subsequences in query sequence $Y$. The six blue small circles shown in Figure 3(b) indicate the six local minima. To determine the indices of the starting point of the six subsequences in query sequence $Y$, the optimal warping path is computed in reverse order of indices starting with each six local minima. The six resulting "matched subsequences," that is, the subsequences of query $Y$ similar to reference $X$, are shown in Figures 4(a) and 4(b). In the next iteration, using classic DTW, we compute the six final accumulated cost matrices, as shown in Figure 4(a), and finally, normalization of the distance (see Figure 4(b)) between the reference and the matched six subsequences in query is conducted.

\section{Results and Discussion}

For our time sequence data sets, we only employed the $x$ axis acceleration data to calculate distance (DTW distance) by using the aDTW algorithm described in Section 2. We iteratively found the 36 matched subsequences for all six successive rounds, but not all participant data showed good matching. The numbers of matched subsequences for the four participants are $35,35,29$, and 31 . One of the 36 subsequences was a reference, and 35 was the maximum number of matched subsequences found automatically. 


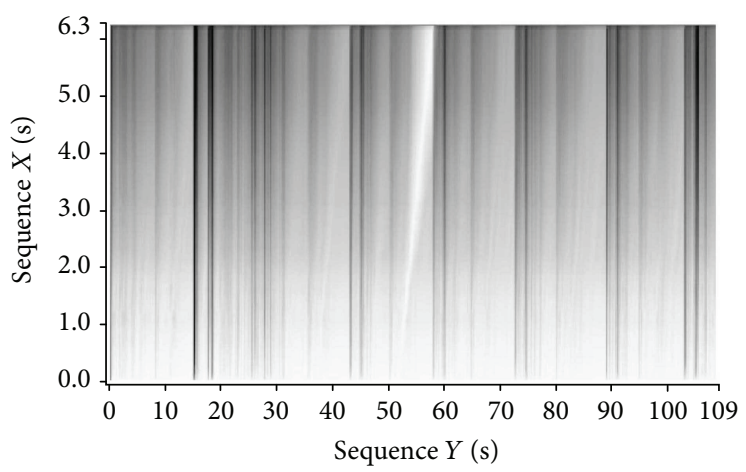

(a)

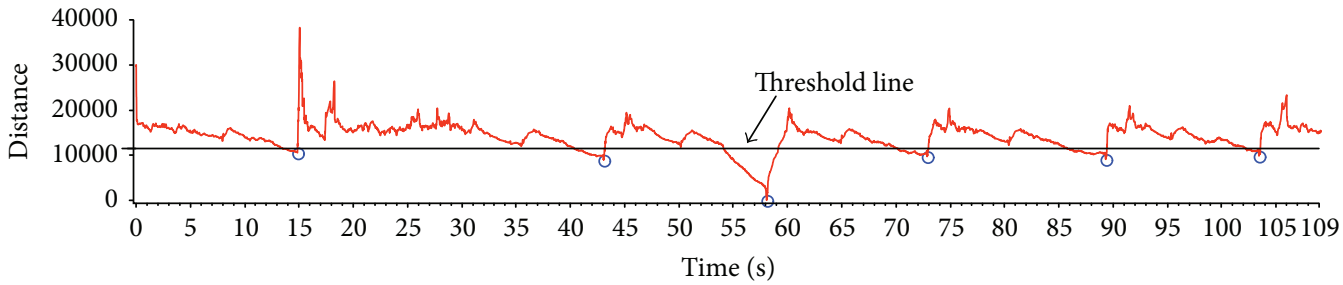

(b)

Figure 3: (a) Accumulated cost matrix between sequences $X$ and $Y$ for calculating distance function. Regions of low cost are indicated by light colors, and those of high cost are indicated by dark colors. (b) Distance function that corresponds to top row of accumulated cost matrix. Blue small circles indicate six local minima with distance below threshold. Indices of six local minima correspond to end point of six subsequences in $Y$.

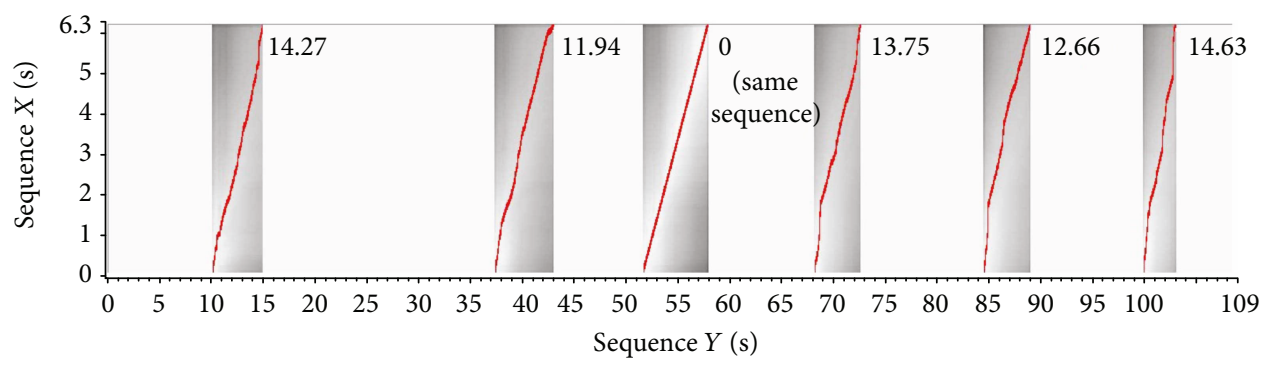

(a)
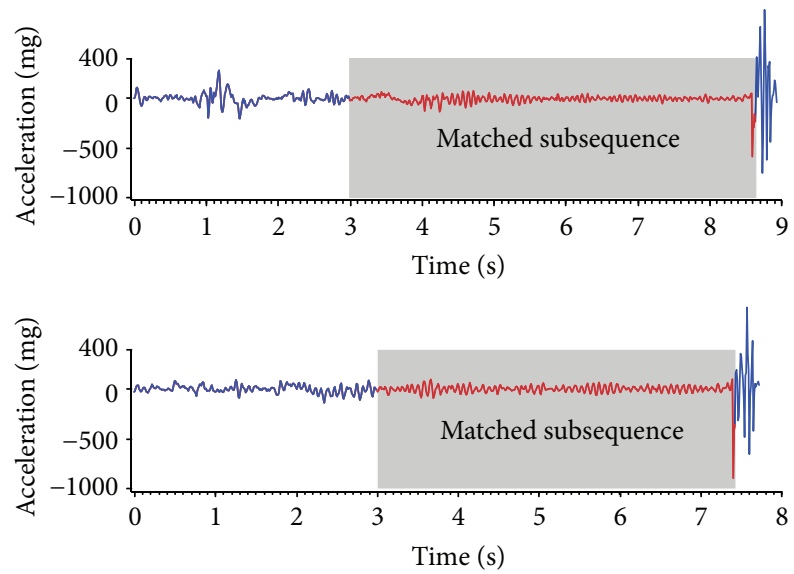

(b)

FIGURE 4: (a) Final accumulated cost matrix between sequence $X$ and six matched subsequences in $Y$. Regions of low cost are indicated by light colors, and those of high cost are indicated by dark colors. Six grey regions correspond to six matched subsequences in $Y$. Grey (red in color print) lines indicate six optimal warping paths. Value indicates normalized distance between sequence $X$ and each matched subsequences. (b) Resulting two (correspond to the second and fourth subsequences) of six subsequences indicated by grey (red in color print) lines. 


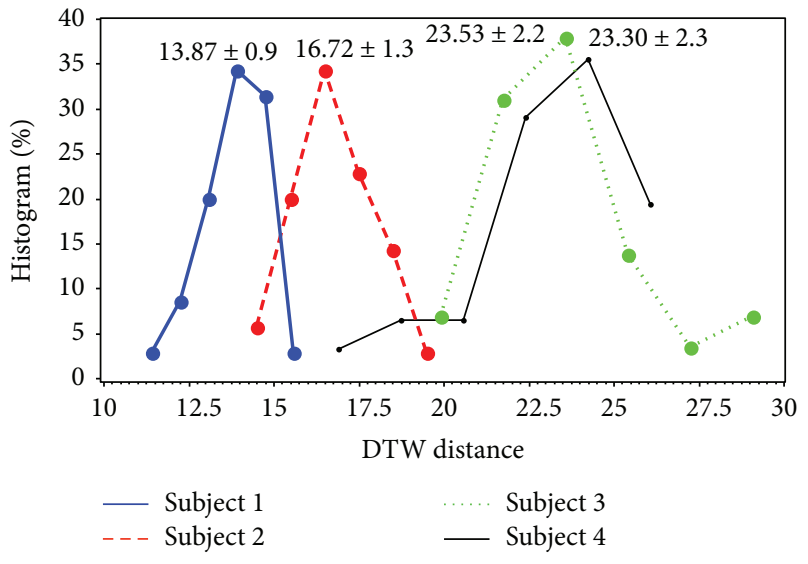

Figure 5: Histogram of distance (DTW distance) that corresponds to four participants and relationship between scores and distance (postural consistency).

Then, we calculated the distance between the reference and the subsequence found automatically for the four participants. The mean and SD value of the distance for each participant are as follows: $13.87 \pm 0.9,16.72 \pm 1.3,23.53 \pm 2.2$, and $23.30 \pm 2.3$.

In order to evaluate the relationship between distance and the scores obtained, we plotted a histogram of the DTW distance of each participant. The histogram of DTW distances that correspond to the four participants and the relationship between shooting consistency (distance) and scores are presented in Figure 5. The results show that the higher the score (59 of 60) achieved by an archer, the higher level is the shooting consistency (lower distance, that is, distance of 13.87 and 16.72) that they showed. Subjects 1 and 2 are female archers with relatively higher score. A small distance value indicates that an archer has maintained highlevel shooting consistency while archery shooting repetitively. On the other hand, the lower the score (54 of 60 and 55 of 60) achieved by an archer, the lower level is the shooting consistency (higher distance, that is, distance of 23.53 and 23.30) that they showed. Subjects 3 and 4 are female archers with relatively lower score.

In this study, we provide a useful signal processing method that quantifies shooting consistency in archery. The results show that the DTW algorithm is a very useful metric for assessing shooting skills, evaluating the progress on training, and finding talented archers.

\section{Conclusions}

In this study, we measured four $(n=4)$ elementary level archers from a middle school in Korea for the purpose of analyzing shooting consistency in archery. Such shooting consistency is defined by the distance between two time sequences with respect to acceleration data. The distance was calculated using the DTW algorithm. The results showed that the higher the scores achieved by an archer, the lower is the distance, that is, the higher level is the shooting consistency showed by the archer.
Consequently, distance can be used as a quantitative parameter for measuring similarity of movement, which is, shooting consistency in archery. Moreover, the DTW algorithm-based approach provides the advantage of searching all matched subsequences automatically from a long sequence. We inferred that the proposed approach might be important for assessing shooting skills, evaluating an archer's progress, and finding talented archers in advance. The ultimate goal of this study is to develop measures to identify archers' talents and using these measures to improve archers' performance and preventing injuries ahead.

\section{Conflicts of Interest}

The authors declare no conflict of interest.

\section{Authors' Contributions}

Cheng-Hao Quan and Sangmin Lee conceived and designed the experiments. Cheng-Hao Quan and Sangmin Lee performed the experiments. Cheng-Hao Quan analyzed the data and prepared the figures. Cheng-Hao Quan drafted the paper. Cheng-Hao Quan, Zia Mohy-Ud-Din, and Sangmin Lee revised and approved the final version of the paper.

\section{Acknowledgments}

This research was supported by the Basic Science Research Program through the NRF (National Research Foundation of Korea) funded by the Ministry of Education (2010-0020163).

\section{References}

[1] J. Stuart and J. Atha, "Postural consistency in skilled archers," Journal of Sports Sciences, vol. 8, no. 3, pp. 223-234, 1990.

[2] H. Ertan, "Muscular activation patterns of the bow arm in recurve archery," Journal of Science and Medicine in Sport, vol. 12, no. 3, pp. 357-360, 2009.

[3] B. Horsak and M. Heller, "A three-dimensional analysis of finger and bow string movements during the release in archery," Journal of Applied Biomechanics, vol. 27, pp. 151-160, 2011.

[4] D. L. Mann and N. Littke, "Shoulder injuries in archery," Canadian Journal of Sport Sciences, vol. 1989, no. 14, pp. 8592, 1989.

[5] P. Leroyer, J. Van Hoecke, and J. N. Helal, "Biomechanical study of the final push-pull in archer," Journal of Sports Sciences, vol. 11, no. 1, pp. 63-69, 1993.

[6] A. Nishizono, H. Shibayama, T. Izuta, and K. Saito, Analysis of Archery Shooting Techniques by Means of Electromyography, J. Tsarouchas, J. Terauds, B. A. Gowitzke and L. E. Holt, Eds., International symposium on biomechanics in sports, Athens, Greece, 1987.

[7] E. Keogh and A. Ratanamahatana, Everything You Know About Dynamic Time Warping Is Wrong, Workshop on Mining Temporal and Sequential Data, in conjunction with 10th ACM SIGKDD Int. Conf. Knowledge Discovery and Data Mining (KDD-2004), Seattle, WA, USA, 3rd edition, 2004.

[8] F. Itakura, "Minimum prediction residual principle applied to speech recognition," in IEEE Transactions on Acoustics, Speech, and Signal Processing, pp. 52-72, IEEE, New York, NY, USA, 1975. 
[9] M. H. Ko, G. West, S. Venkatesh, and M. Kumar, "Using dynamic time warping for online temporal fusion in multisensor systems," Information Fusion, vol. 9, no. 3, pp. 370-388, 2008.

[10] J. Liu, L. Zhong, J. Wickramasuriya, and V. Vasudevan, "Uwave: accelerometer-based personalized gesture recognition and its applications," Pervasive and Mobile Computing, vol. 5, no. 6, pp. 657-675, 2009.

[11] N. Gillian, R. B. Knapp, and S. O’Modhrain, "Recognition of multivariate temporal musical gestures using $\mathrm{N}$-dimensional dynamic time warping," in The 11th Int'l conference on New Interfaces for Musical Expression, Oslo, Norway, 2011.

[12] E. Keogh and A. C. Ratanamahatana, "Exact indexing of dynamic time warping," Knowledge and Information Systems, vol. 7, pp. 358-386, 2004. 


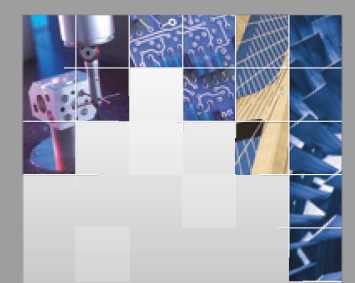

\section{Enfincering}
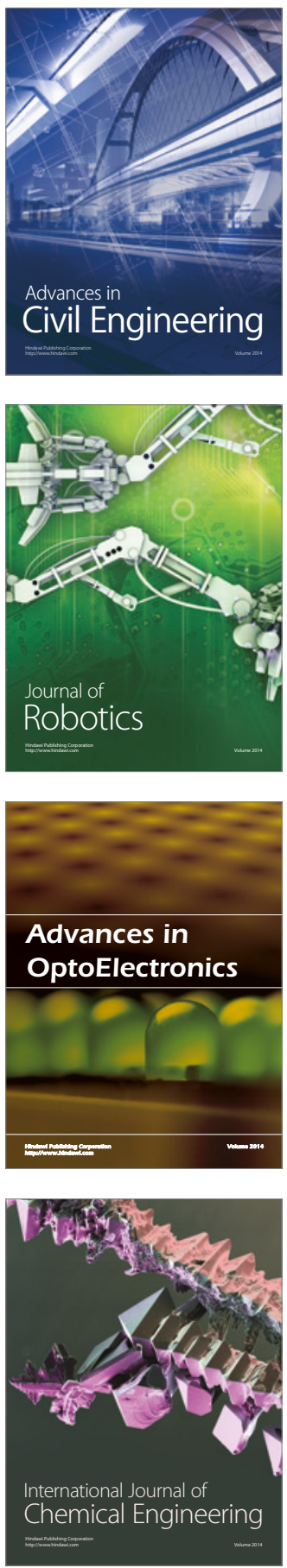

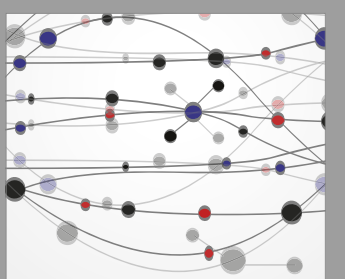

The Scientific World Journal

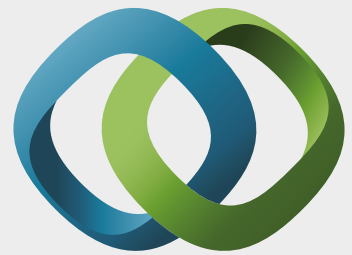

\section{Hindawi}

Submit your manuscripts at

https://www.hindawi.com
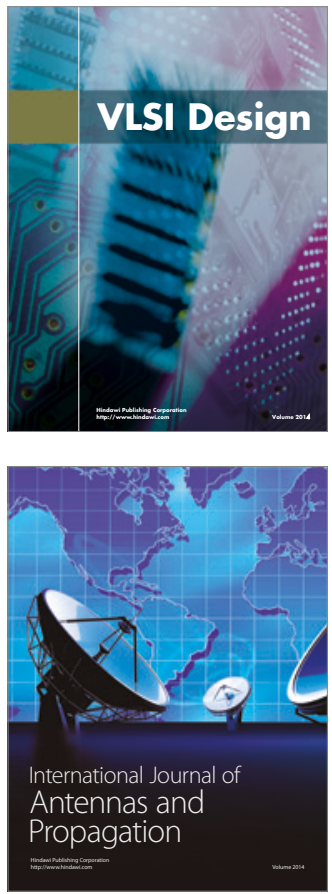

\section{Rotating}

Machinery
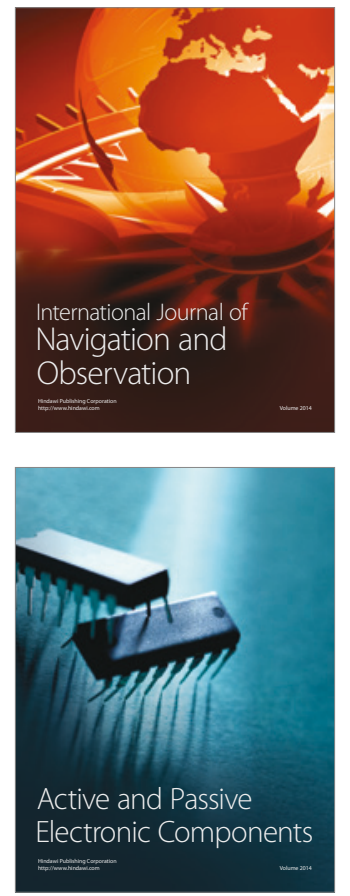
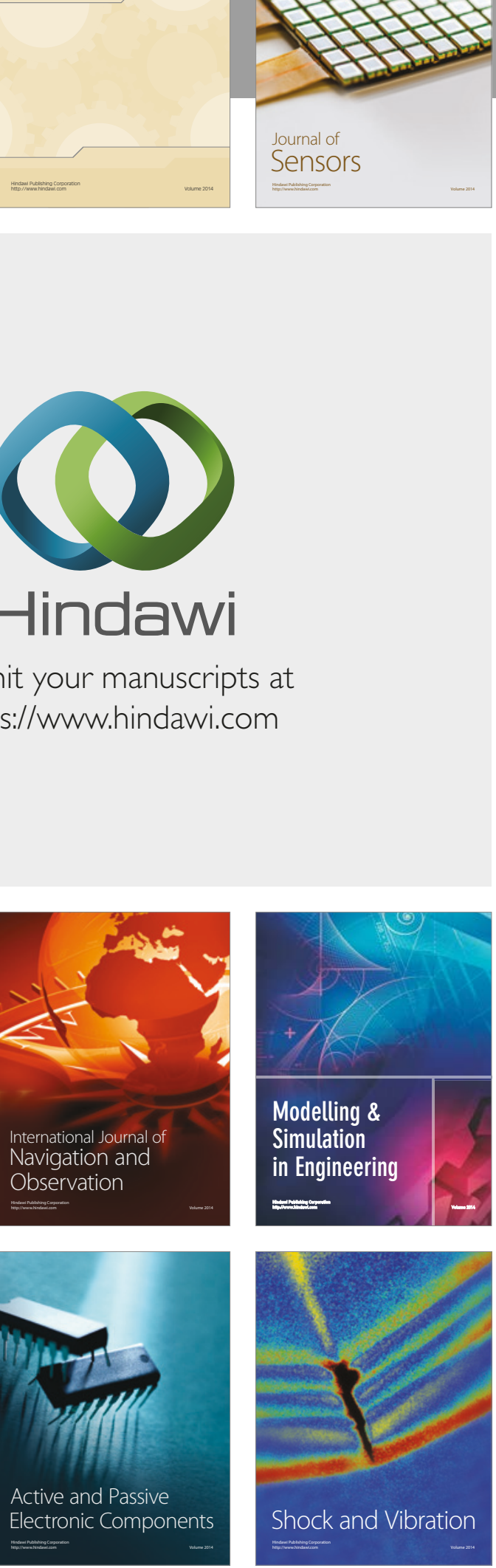
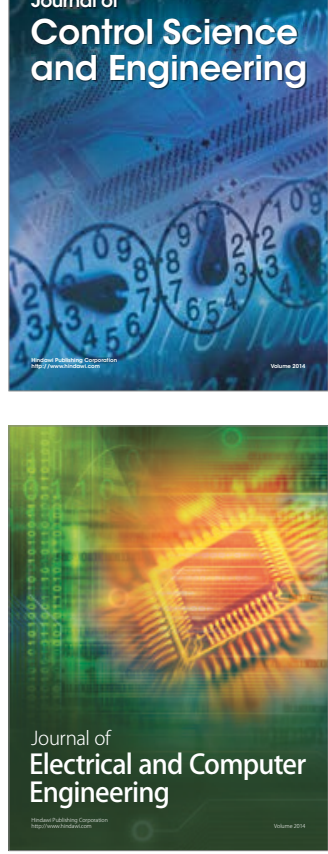

Distributed

Journal of

Control Science

and Engineering
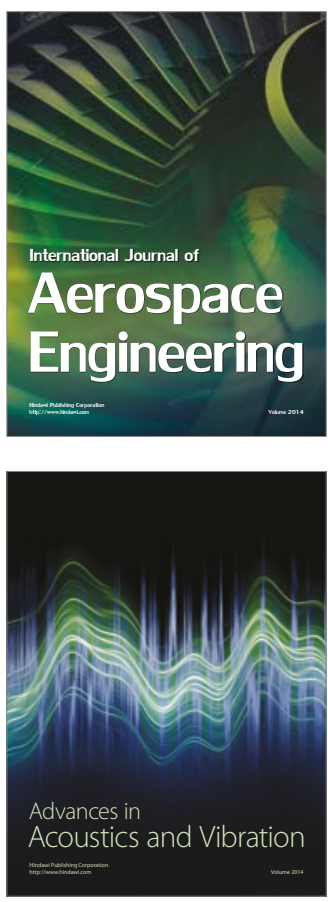

Sensor Networks 